\title{
The Significance of the Mediterranean Diet in the Management of Non-Alcoholic Fatty Liver Disease: A Systematic Review
}

Harpreet Gosal ${ }^{1,2}$, Harsimran Kaur ${ }^{3}$, Hyginus Chakwop Ngassa ${ }^{4,5}$, Khaled A. Elmenawi ${ }^{6,7}$, Vishwanath Anil ${ }^{1}$, Lubna Mohammed ${ }^{1}$

1. Internal Medicine, California Institute of Behavioral Neurosciences \& Psychology, Fairfield, USA 2. Internal Medicine, Government Medical College, Amritsar, IND 3. Medicine, California Institute of Behavioral Neurosciences \& Psychology, Fairfield, USA 4. Gastrointestinal Surgery, Faculty of Medicine and Surgery, University of Brescia, Brescia, ITA 5. Surgery, California Institute of Behavioral Neurosciences \& Psychology, Fairfield, USA 6. Surgery, Cairo University, Cairo, EGY 7. Internal Medicine/Surgery, California Institute of Behavioral Neurosciences \& Psychology, Fairfield, USA

Corresponding author: Harpreet Gosal, gosalharpreet15@gmail.com

\begin{abstract}
Non-alcoholic fatty liver disease (NAFLD) is the accumulation of intrahepatic fat occurring in the absence of alcohol abuse. The fatty changes in the liver are often the beginning of sequelae of complications, potentially causing steatohepatitis, liver cirrhosis, and hepatocellular carcinoma. The Mediterranean diet is not only a way of eating but is considered a lifestyle and primarily consists of a plant-based diet, with olive oil as the primary lipid. In this study, we reviewed the effectiveness of the Mediterranean diet on NAFLD and its efficacy in disease management. This systematic review follows the Preferred Reporting Items for Systematic Reviews and Meta-Analyses Protocol (PRISMA-P) 2009 guidelines. The PubMed database was used to gather articles, using the following terms individually and in combination, "Mediterranean diet," "non-alcoholic fatty liver disease," "insulin resistance," "metabolic syndrome," "omega-3-fatty acids." A quality appraisal was completed to include 14 articles in this systematic review. The studies discuss the correlation between the Mediterranean diet and its role in preventing and treating NAFLD. Olive oil is the major monounsaturated fatty acid, whereas nuts, seeds, and fish consist largely of polyunsaturated fatty acids, both of which are essential components of the Mediterranean diet. The plant-based diet, having sufficient amounts of vegetables, legumes, and fruits, provides its anti-oxidant and anti-inflammatory effects, playing a fundamental role in preventing oxidative stress damage. Olive oil polyphenols increase apoptosis and cell cycle arrest. They also decrease proliferation and angiogenesis, all of which prevent neoplasia. Adapting the Mediterranean lifestyle has shown promising effects in NAFLD, reducing overall mortality and morbidity.
\end{abstract}

Received 05/10/2021

Review began 05/28/2021 Review ended 06/05/2021 Published 06/13/2021

๑) Copyright 2021 Gosal et al. This is an open access article distributed under the terms of the Creative Commons Attribution License CC-BY 4.0., which permits unrestricted use, distribution, and reproduction in any medium, provided the original author and source are credited.

Categories: Internal Medicine

Keywords: nonalcoholic fatty liver disease (nafld), mediterranean diet, nash and steatosis, olive oil, plant-based diet

\section{Introduction And Background}

The diagnosis of non-alcoholic fatty liver disease (NAFLD) is considered when intrahepatic fat accumulation exceeds $5 \%$ in the absence of alcohol abuse [1]. The disease prevalence is estimated to be a quarter of the world population, with a continuing rise [2]. Currently, it is the most common cause of abnormal liver tests in developed countries, with a prevalence of $46 \%$ in the United States [3,4]. The occurrence of fatty changes is only the beginning of the potentially life-threatening complications occurring over time, including steatohepatitis, liver cirrhosis, and hepatocellular carcinoma [1]. NAFLD is the leading cause of liver transplantation in men and the second leading cause in women [5]. The manifestations from NAFLD cause sustained liver damage and negatively affected the cardiovascular system, increasing overall mortality, and morbidity [5].

The Mediterranean diet dates back to the traditional dietary patterns from the Mediterranean regions, which are considered a virtual geographic location consisting of numerous cultures [6,7]. The Mediterranean diet has many variations, but its main emphasis has been a plant-based diet, with significant fiber, anti-oxidants, vegetable proteins, polyunsaturated, and monounsaturated fat [8]. It is known as a high-fat diet with olive oil as the principal source, and the fat content ranging from $35 \%$ to $45 \%$ of the total energy intake [6]. The accumulation of fat occurring due to an imbalance in hepatic lipid metabolism is due to insulin resistance [3]. Hence, insulin resistance is known as the initial step in NAFLD. It is responsible for forming reactive oxygen species (ROS), causing oxidative stress, lipotoxicity, subsequent inflammation, and hepatic damage [3]. The high fruit and vegetable content in the Mediterranean diet has sufficient anti-oxidant and antiinflammatory properties, counteracting the effects of ROS and the pathogenesis of NAFLD [3]. A similar effect has been observed with olive oil, decreasing inflammation by inhibiting transcription genes [6]. The anti-oxidant effects of the Mediterranean diet seem to share some form of inverse correlation with the underlying pathology of NAFLD. 


\section{Cureus}

Research shows that several diets have been tested for NAFLD with various effects. The question remains, is the Mediterranean diet a potential treatment and preventive measure for NAFLD in the near future? It is also the weight loss in response to the diet that significantly affects the disease progression, or is it the ingredients that make the Mediterranean diet that play an essential role [3]? The current treatment recommendations for patients with NAFLD are explored in this article, focusing on how effective the Mediterranean diet is in disease management. Also, we analyze whether adopting the Mediterranean diet early in the steatosis stage of the disease results in any alteration in disease outcome. The fundamental aim of this review is to report the current evidence about the effects of the Mediterranean diet in NAFLD patients and translate this main dietary recommendation to guidelines for clinical practice for disease prevention and treatment.

\section{Review}

This section will discuss how articles were gathered and reviewed for this systematic review. The review analyzes the significance of monosaturated and polyunsaturated fats, the anti-inflammatory effects of the Mediterranean diet, the benefits of limiting sodium, and how these factors alter the outcome of NAFLD.

\section{Methods}

The protocol of this systematic review follows the Preferred Reporting Items for Systematic Reviews and Meta-Analyses Protocol (PRISMA-P) 2009 guideline. The electronic database PubMed was searched for articles. The keywords and medical subject headings $(\mathrm{MeSH})$ terms were used individually and in combination to identify relevant articles. The keywords and MeSH terms included in the search strategy were "Mediterranean diet," "non-alcoholic fatty liver disease," "insulin resistance," "metabolic syndrome," and "omega-3-fatty acids." A total of 1108 publications were checked for duplications and assessed for relevance to the research topic. The articles were then screened using the inclusion and exclusion criteria to identify the eligibility of the studies. Table 1 and Table 2 summarize the search strategy using MeSH terms and keywords.

\begin{tabular}{|c|c|c|c|}
\hline Keyword & Database & Before inclusion/exclusion & After inclusion/exclusion \\
\hline Insulin resistance [MeSH] AND fatty steatosis [MeSH] & PubMed & 1056 & 136 \\
\hline Mediterranean diet [MeSH] & PubMed & 341 & 79 \\
\hline NAFLD [MeSH] AND omega-3-fatty acids [MeSH] & PubMed & 101 & 34 \\
\hline NAFLD [MeSH] AND Mediterranean diet [MeSH] & PubMed & 4 & 4 \\
\hline Insulin resistance [MeSH] AND Mediterranean diet [MeSH] & PubMed & 6 & 0 \\
\hline
\end{tabular}

TABLE 1: Search strategy with MeSH terms

MeSH: medical subject headings; NAFLD: non-alcoholic fatty liver disease

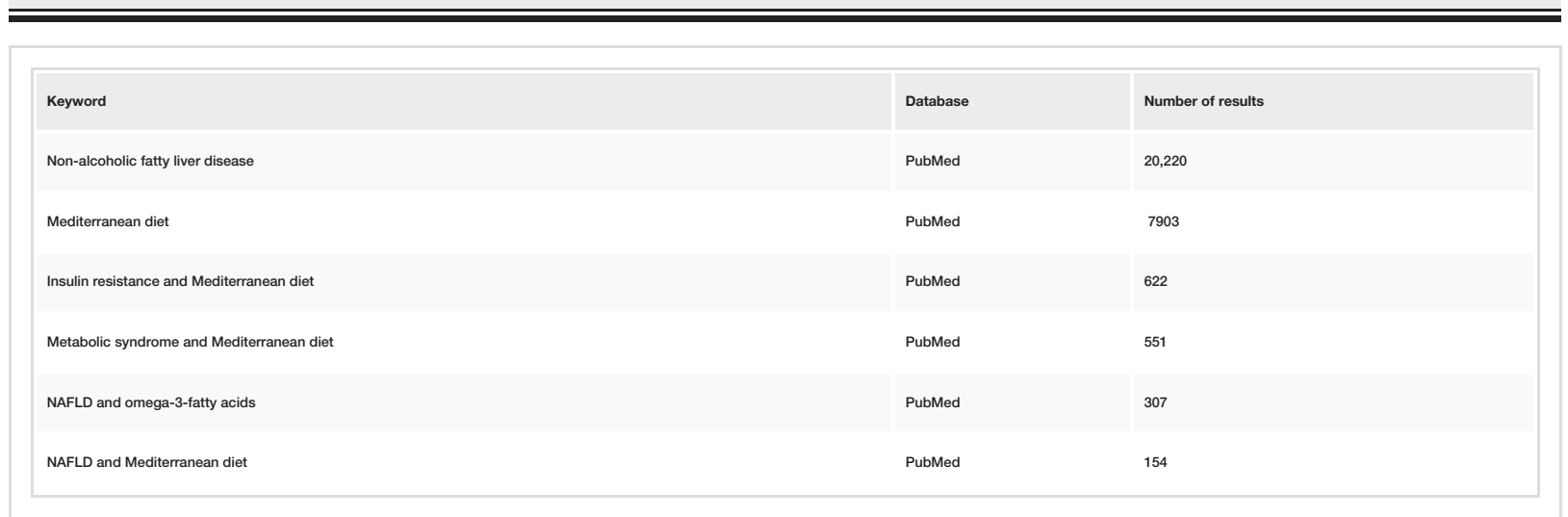

TABLE 2: Search strategy with keywords 
only, studies comprised a mixture of systematic reviews, observational (case-control, cohort, and crosssectional studies), meta-analysis, and literature review were included. The animal studies and articles with a publication date more than five years ago were excluded.

\section{Results}

A total of 1108 publications were identified from PubMed, from which 526 duplicates were removed. Filters were applied based on inclusion criteria (full-text studies in English, last five years, on humans, all types of reviews), yielding 274 articles. Each article was manually reviewed for its content, and articles irrelevant to the study topic were excluded. The eligibility of the remaining articles was checked using the PRISMA checklist, Joanna Briggs Institute (JBI) checklist, New Castle Ottawa Tool (developed as a collaboration between the University of Newcastle, Australia, and the University of Ottawa, Canada), and a measurement tool to assess systematic reviews (AMSTAR) checklist. A total of 14 studies were ultimately selected and included in the qualitative synthesis. Figure 1 below shows the PRISMA flowchart diagram of literature retrieval.

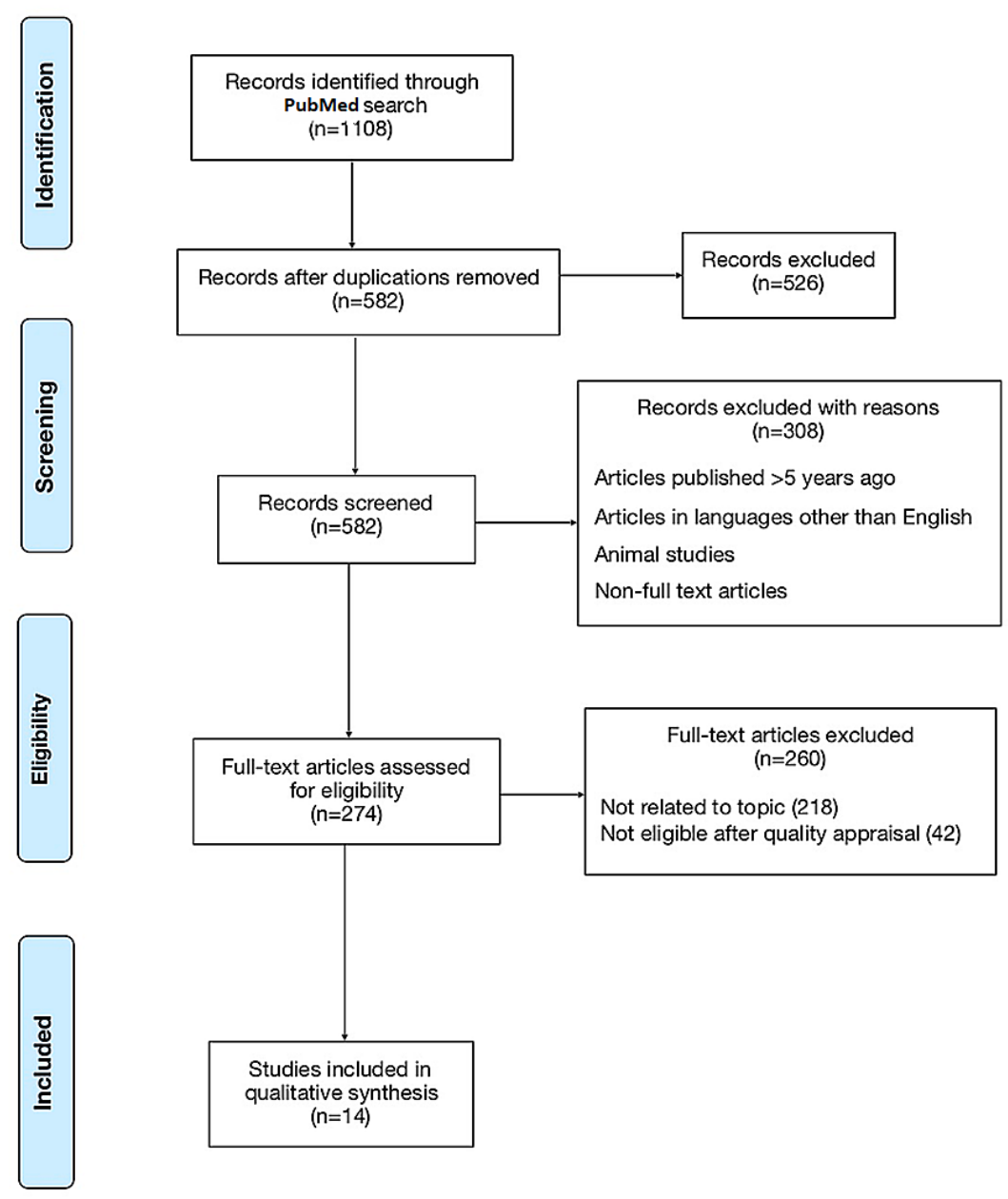

\section{FIGURE 1: PRISMA flow diagram}

PRISMA: Preferred Reporting Items for Systematic Review and Meta-Analysis

\section{Discussion}

Figure 2 below shows the Mediterranean diet food pyramid with daily, weekly, and monthly consumptions [5]. 


\section{Cureus}

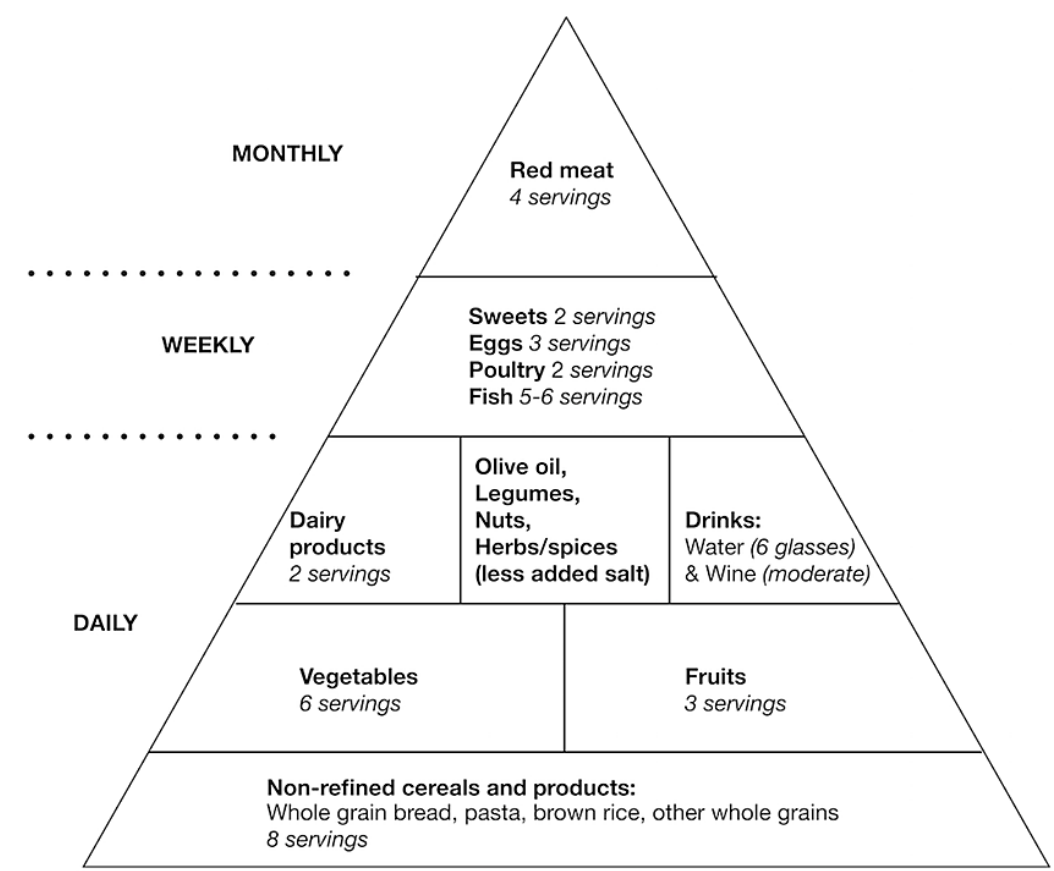

FIGURE 2: Mediterranean diet pyramid

Daily

The daily servings consist of high consumption of non-refined cereals and grain products, and vegetables and fruits, along with pulses, legumes, and nuts [9]. Olive oil is the main lipid and considered an essential part of the Mediterranean diet. Also, salt intake is limited by replacing herbs and spices at the table [7]. Besides, adequate water intake, approximately six glasses, is ideal, along with wine in moderation. The consumption of red wine with meals dates back to the traditional Mediterranean diet of the European region [7].

Weekly

Eggs, poultry, and fish are limited to weekly intake, with fish having the highest recommended servings of five or six. Sweets are often limited to a maximum of two servings in the week.

Monthly

The Mediterranean diet also focuses on reducing red meat consumption, with only four servings per month [10]. As mentioned above, the main focus of fat is switched over to olive oil. Hence, red meat intake is significantly decreased.

\section{Olive oil and the role of monounsaturated fat}

Olive oil is the most representative group of the Mediterranean diet, and oleic acid, a monounsaturated fatty acid (MUFA), is a major component of this essential dietary fat [5]. MUFAs have numerous beneficial effects, including healthy blood lipid profiles, lowering blood pressure, and improving insulin sensitivity which is an essential and first step in preventing NAFLD [3]. Monounsaturated and polyunsaturated fatty acid (PUFA) diets reduce fasting plasma triacylglycerol and very-low-density lipoprotein (VLDL)-cholesterol concentration on average $19 \%$ and $22 \%$, respectively [11]. They also cause an increase in high-density lipoprotein (HDL)-cholesterol levels, which is highly beneficial as current research emphasizes higher HDL levels as preventive measures for long-term diseases [12]. The studies indicate a cardiovascular risk reduction and an all-case mortality reduction linked to the benefits of olive oil [13]. The long-standing conditions show a close relationship to NAFLD prevalence, for example, with over $50 \%$ of cases occurring in type 2 diabetes mellitus (T2DM) [14]. The large intake of MUFAs as a substitute for trans fatty acids is believed to reduce the risk of T2DM in high-risk patients by up to $83 \%$ over a median of 4.4 years [14]. NAFLD is limited to insulin resistance and includes a spectrum of liver conditions associated with intrahepatic fat accumulation and further complications. For example, a quarter of NAFLD patients progress to non-alcoholic steatohepatitis, which is also associated with a higher risk of cardiovascular disease and mortality [14]. In conclusion, a healthy lipid panel is essential in eliminating countless long-standing health concerns. Monounsaturated fatty acids, driven from olive oil, seem to play an outstanding role in preventive 
medicine.

\section{Nuts and seeds and the role of polyunsaturated fatty acid}

Asides from oil olive as the main source of MUFA in the Mediterranean diet, high quantities of PUFAs in nuts, seeds, and fish also play a valuable role [15]. The most important PUFA for NAFLD is long-chain n-3 fatty acids, which include eicosapentaenoic, EPA, and docosahexaenoic, DHA [15]. These prevent liver adipogenesis by modifying transcription and suppressing peroxisome proliferator-activated receptor $\alpha$ and sterol regulatory element-binding protein-1 genes [16]. The main role of these genes is the regulation of hepatic fatty acid oxidation and synthesis, respectively [16]. Additionally, n-3 fatty acids suppress the direct uptake of fat into hepatocytes and modulate the expression of particular miRNAs [16,17]. The wellestablished outcome of PUFA and NAFLD by research is the prevention of hepatic steatosis, resulting in a beneficial role in disease management [18].

\section{Effects of olive oil and a plant-based diet on inflammatory-related mechanisms}

A bulk of the Mediterranean diet comprises plant-based foods, with a focus on plant-based protein sources. This includes legumes, moderate consumption of fish, and a limited amount of red meat [13]. Vegetables, fruits, and legumes are high in anti-oxidants and anti-inflammatory, playing a fundamental role in preventing oxidative stress damage and cytokine release [13]. From these food sources, legumes have been identified as the most predictive component for lowering mortality [13]. Vegetables and fruits are equally important and consist of polyphenols, which are important bioactive compounds that increase fatty acid oxidation [7]. Polyphenols promote fatty acid beta-oxidation and exert their anti-inflammatory role by inhibiting NF-KB directly or via peroxisome proliferator-activated receptors (PPARs), and chronic inflammatory pathways such as prostaglandin E2 (PGE2) and cyclooxygenase-2 (COX-2) [15]. The recent literature has also shown a relationship between the ability of polyphenols to module the immune system by altering the role of WBCs and inflammatory cytokines that participate in the immunological defense [19]. Hence, the Mediterranean diet has been shown to positively influence inflammatory biomarkers, including adhesion molecules and cytokines, in presenting hepatic fat accumulation and ensuring limited blood levels of transaminase, triglycerides, cholesterol, and insulin resistance [20]. In conclusion, olive oil and plantbased diets, which are rich in polyphenols, have shown to have a positive impact on the human body's defense mechanism.

\section{Anti-cancer mechanism from olive oil polyphenols}

NAFLD is associated with an increased risk of numerous cancers, with a well-established relationship between NAFLD and hepatocellular cancer [21]. However, research has shown that NAFLD is associated with an increased risk for prostate and colon cancer [22]. When comparing the Mediterranean region with other populations, cancer incidence is drastically lower [22]. The role of olive oil and its associated polyphenols, especially oleuropein hydroxytyrosol, is an important component of the underlying anti-cancer mechanism [23]. Polyphenols increase apoptosis and cell cycle arrest while decreasing proliferation and angiogenesis [22]. The role of apoptosis is indicative of the increase in cell death domains, such as caspase-3,-7, p53, and Bax and Bak [22]. At the same time, suppression of neovascularization by olive oil polyphenols has resulted in a decrease in growth factors such as VEGF and TGFalpha-induced migration of hepatocellular carcinoma cells [21]. These mechanisms drastically inhibited the underlying pathology and worked in preventing malignancy.

\section{Limiting sodium}

The Mediterranean diet aims to replace salt at the table with herbs and spices, ensuring favor yet adequate blood pressure control. The relationship between high sodium levels and blood pressure is well established, but the direct effect on NAFLD is controversial. The current World Health Organization (WHO) guidelines indicate a sodium intake of $<2000 \mathrm{mg} / \mathrm{dl}$ [24]. Many epidemiological studies have proven to show a positive correlation between sodium intake and body weight, independent of energy or sugar intake [24]. The literature has established a relationship between increase sodium intake and triglyceride levels, indicating a potential risk factor for NAFLD [24]. The recommendation of a low-sodium diet has been beneficial in lowering overall mortality and morbidity, with hypertension being an underlying cause of many longstanding conditions [25].

Table 3 summarizes experimental and observational studies that were reviewed, and the study group analyzed along with the findings. These studies involved smaller populations but illustrated a positive correlation between NAFLD and the Mediterranean diet in numerous ways. This includes the reduction of intrahepatic fat accumulation and steatosis in relation to consuming the Mediterranean diet and an overall positive impact on NAFLD score. 


\section{Cureus}

\begin{tabular}{|c|c|c|c|c|}
\hline References & Study type & Study characteristics & Method & Outcome \\
\hline $\begin{array}{l}\text { Biolato et } \\
\text { al., } 2019 \\
{[18]}\end{array}$ & Crossover & $\begin{array}{l}90 \text { Caucasian patients, } \\
90 \% \text { male, median age } 43 \\
\text { years, body mass index } \\
309 \text { with NAFLD (confirmed } \\
\text { by biopsy) }\end{array}$ & $\begin{array}{l}\text { Participants underwent } 16 \text { weeks of the Mediterranean diet, } 16 \text { weeks of a free wash- } \\
\text { out, and } 16 \text { weeks of the low-fat diet }\end{array}$ & $\begin{array}{l}\text { Mediterranean diet can improve intestinal permeability in patients with } \\
\text { NAFLD, hence is effective in treating visceral obesity and elevated serum } \\
\text { transaminase }\end{array}$ \\
\hline $\begin{array}{l}\text { Mirizzi et } \\
\text { al., } 2019[9]\end{array}$ & $\begin{array}{l}\text { Cross- } \\
\text { sectional } \\
\text { study }\end{array}$ & 136 participants & $\begin{array}{l}\text { Diagnosis of NAFLD was determined using vibration-controlled elastography on a } \\
\text { FibroScan and categorized as either NAFLD absent (<215 dB), mild (215-250 dB), and } \\
\text { severe ( }>300 \mathrm{~dB})\end{array}$ & $\begin{array}{l}\text { A greater number of participants that were overweight, obese, and with a } \\
\text { higher waist circumference fell into the Severe NAFLD category }\end{array}$ \\
\hline $\begin{array}{l}\text { Franco et } \\
\text { al., } 2020[2]\end{array}$ & $\begin{array}{l}\text { Observational } \\
\text { study: } \\
\text { randomized } \\
\text { clinical trial }\end{array}$ & $\begin{array}{l}144 \text { moderate or severe } \\
\text { NAFLD patients }\end{array}$ & $\begin{array}{l}\text { Participants were assigned six interventions arms during three months, which } \\
\text { included control diet, low glycemic index Mediterranean diet, aerobic activity } \\
\text { program, combined activity program, low glycemic index Mediterranean diet plus } \\
\text { aerobic activity program, or low glycemic index Mediterranean diet plus combined } \\
\text { activity program }\end{array}$ & $\begin{array}{l}\text { NAFLD score significantly reduced after } 45 \text { days of treatment in every } \\
\text { working arm except for the control diet group }\end{array}$ \\
\hline $\begin{array}{l}\text { Yaskolka et } \\
\text { al., } 2020[1]\end{array}$ & $\begin{array}{l}\text { Observational } \\
\text { study: } \\
\text { randomized } \\
\text { clinical trial }\end{array}$ & $\begin{array}{l}294 \text { participants with } \\
\text { abdominal } \\
\text { obesity/dyslipidemia }\end{array}$ & $\begin{array}{l}\text { Participants assigned into one of the three groups: healthy dietary guidelines, } \\
\text { Mediterranean diet, or green-Mediterranean weight-loss diet, all accompanied by } \\
\text { physical activity }\end{array}$ & $\begin{array}{l}\text { NAFLD prevalence declined with the Mediterranean diet, and after } 18 \\
\text { months, both Mediterranean groups had significantly higher total plasma } \\
\text { polyphenols. Also, intra-hepatic fraction percentage loss was seen with } \\
\text { Mankai and walnuts intake and decreased red/processed meat } \\
\text { consumption }\end{array}$ \\
\hline $\begin{array}{l}\text { Campanella } \\
\text { et al., } 2020 \\
\text { [8] }\end{array}$ & $\begin{array}{l}\text { Observational } \\
\text { study: } \\
\text { randomized } \\
\text { clinical trial }\end{array}$ & 556 participants & $\begin{array}{l}\text { Participants were assigned to one of three diets (Mediterranean diet, low glycemic } \\
\text { Mediterranean diet, low glycemic index Mediterranean diet) or the control group. } \\
\text { Anthropometric variables were noted for each participant, and the outcome of NAFLD } \\
\text { was observed }\end{array}$ & $\begin{array}{l}\text { Low glycemic index Mediterranean diet is more effective than other diets } \\
\text { in reducing elevated fasting remnant cholesterol }\end{array}$ \\
\hline $\begin{array}{l}\text { Baratta et } \\
\text { al., } 2020[4]\end{array}$ & Cohort study & 238 participants & $\begin{array}{l}\text { Liver ultrasound and Mediterranean-diet questionnaire was done, along with serum } \\
\text { sNox2-dp activity, and serum lipopolysaccharide levels measured for each participant }\end{array}$ & $\begin{array}{l}\text { Out of } 238 \text { participants, } 193(81.1 \%) \text { had liver steatosis, with results } \\
\text { suggesting that the Med-Diet improves redox status and can be a } \\
\text { beneficial approach in NAFLD }\end{array}$ \\
\hline
\end{tabular}

TABLE 3: Observational and experimental studies illustrating the relationship between NAFLD and the Mediterranean diet

NAFLD: non-alcoholic fatty liver disease

Table 4 summarises the current literature reviews that were used in this study to conclude further and establish the effects of the Mediterranean diet on NAFLD. These studies analyzed the risk factors that were influenced by the Mediterranean diet, lowering the outcomes of NAFLD. 


\section{Cureus}

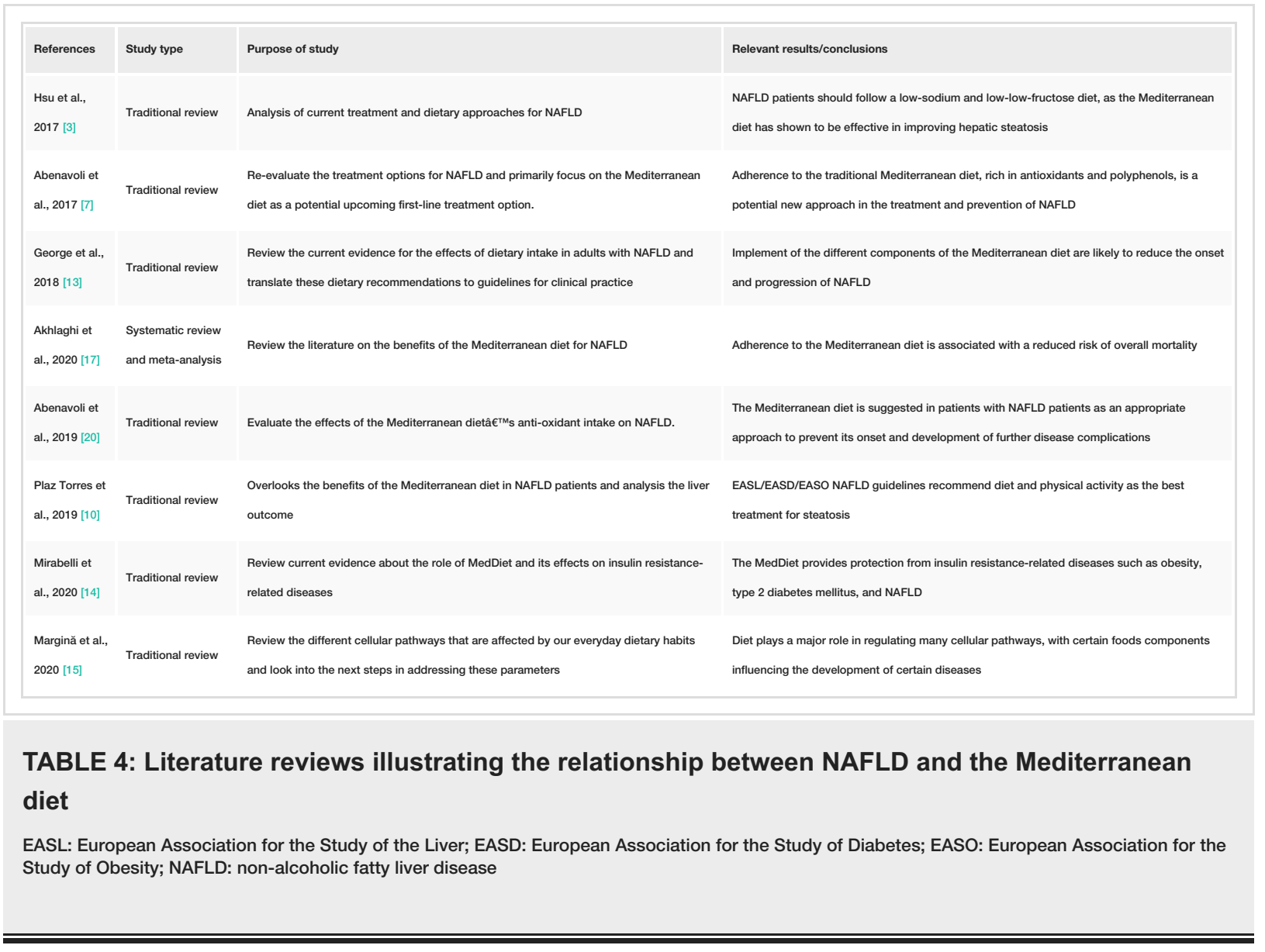

Limitations

This review only includes studies in the English language. As a result, we may have missed studies published in other proficiencies, such as languages dominant in the Mediterranean regions, limiting the definition of the traditional Mediterranean diet. A publication date within the last five years may also cause a similar limitation. The studies prior to 2016 are excluded, while some may have provided valuable information regarding past dietary measures and established relationships to NAFLD. Most of the studies in this review consist of relatively small sample sizes, which potentially limit the efficacy of the results published. Another limitation is the potential confounders that exist among the studies included in this systematic review. This allows for bias, as confounding factors within studies can alter the overall outcome. Lastly, studies use different methods for measuring NAFLD severity, but the definition of Mediterranean diet varied; hence the intervention amongst the studies may not have been consistent.

\section{Conclusions}

This systematic review outlines the significance of the Mediterranean diet in the management of NAFLD and identifies what has been currently studied, and essentially what needs to be focused on in the future to strengthen this relationship. In this review, observational studies, cross-sectional, crossover, and literature reviews were analyzed, indicating a visible link between the two variables. The Mediterranean diet is a regime and a type of lifestyle that has been shown to positively enhancing well-being. It provides a significant number of advantages, being complex in MUFAs, PUFAs, and polyphenols, indicating its antiinflammatory and anti-cancer mechanisms. After reviewing the literature, the Mediterranean diet can be considered a major step forward in NAFLD management. It promotes weight loss by replacing saturated fats and carbohydrates and plays a direct role in eliminating NAFLD pathology. Adapting the Mediterranean diet in the early stages may result in alterations in disease outcome, but more studies are required to confirm this correlation. In the near future, along with more extensive studies, we may potentially be able to conclude the Mediterranean diet as the first-line preventive and treatment option for NAFLD.

\section{Additional Information}

\section{Disclosures}

Conflicts of interest: In compliance with the ICMJE uniform disclosure form, all authors declare the following: Payment/services info: All authors have declared that no financial support was received from any organization for the submitted work. Financial relationships: All authors have declared that they have no financial relationships at present or within the previous three years with any organizations that might 
have an interest in the submitted work. Other relationships: All authors have declared that there are no other relationships or activities that could appear to have influenced the submitted work.

\section{References}

1. Yaskolka Meir A, Rinott E, Tsaban G, et al.: Effect of green-Mediterranean diet on intrahepatic fat: the DIRECT PLUS randomised controlled trial. [In Press]. Gut. 2021, 10.1136/gutinl-2020-323106

2. Franco I, Bianco A, Mirizzi A, et al.: Physical activity and low glycemic index Mediterranean diet: main and modification effects on NAFLD score. Results from a randomized clinical trial. Nutrients. 2020, 13:66. 10.3390/nu13010066

3. Hsu CC, Ness E, Kowdley KV: Nutritional approaches to achieve weight loss in nonalcoholic fatty liver disease. Adv Nutr. 2017, 8:253-265. 10.3945/an.116.013730

4. Baratta F, Pastori D, Bartimoccia S, et al.: Poor adherence to Mediterranean diet and serum lipopolysaccharide are associated with oxidative stress in patients with non-alcoholic fatty liver disease. Nutrients. 2020, 12:1732. 10.3390/nu12061732

5. Angelico F, Ferro D, Baratta F: Is the Mediterranean diet the best approach to NAFLD treatment today? . Nutrients. 2021, 13:739. 10.3390/nu13030739

6. Pérez-Martínez P, Mikhailidis DP, Athyros VG, et al.: Lifestyle recommendations for the prevention and management of metabolic syndrome: an international panel recommendation. Nutr Rev. 2017, 75:307-326. 10.1093/nutrit/nux014

7. Abenavoli L, Milic N, Luzza F, Boccuto L, De Lorenzo A: Polyphenols treatment in patients with nonalcoholic fatty liver disease. J Transl Int Med. 2017, 5:144-147. 10.1515/jtim-2017-0027

8. Campanella A, Iacovazzi PA, Misciagna G, et al.: The effect of three Mediterranean diets on remnant cholesterol and non-alcoholic fatty liver disease: a secondary analysis. Nutrients. 2020, 12:1674. 10.3390/nu12061674

9. Mirizzi A, Franco I, Leone CM, et al.: Effects of some food components on non-alcoholic fatty liver disease severity: results from a cross-sectional study. Nutrients. 2019, 11:2744. 10.3390/nu11112744

10. Plaz Torres MC, Aghemo A, Lleo A, et al.: Mediterranean diet and NAFLD: what we know and questions that still need to be answered. Nutrients. 2019, 11:2971. 10.3390/nu11122971

11. Garg A: High-monounsaturated-fat diets for patients with diabetes mellitus: a meta-analysis . Am J Clin Nutr. 1998, 67:577-582. 10.1093/ajcn/67.3.577S

12. Kosmas CE, Martinez I, Sourlas A, et al.: High-density lipoprotein (HDL) functionality and its relevance to atherosclerotic cardiovascular disease. Drugs Context. 2018, 7:212525. 10.7573/dic.212525

13. George ES, Forsyth A, Itsiopoulos C, et al.: Practical dietary recommendations for the prevention and management of nonalcoholic fatty liver disease in adults. Adv Nutr. 2018, 9:30-40.

10.1093/advances/nmx007

14. Mirabelli M, Chiefari E, Arcidiacono B, et al.: Mediterranean diet nutrients to turn the tide against insulin resistance and related diseases. Nutrients. 2020, 12:1066. 10.3390/nu12041066

15. Margină D, Ungurianu A, Purdel C, et al.: Analysis of the intricate effects of polyunsaturated fatty acids and polyphenols on inflammatory pathways in health and disease. Food Chem Toxicol. 2020, 143:111558. 10.1016/j.fct.2020.111558

16. Cansanção K, Citelli M, Carvalho Leite N, López de Las Hazas MC, Dávalos A, Tavares do Carmo MDG, Peres WAF: Impact of long-term supplementation with fish oil in individuals with non-alcoholic fatty liver disease: a double blind randomized placebo controlled clinical trial. Nutrients. 2020, 12:3372. 10.3390/nu12113372

17. Akhlaghi M, Ghasemi-Nasab M, Riasatian M: Mediterranean diet for patients with non-alcoholic fatty liver disease, a systematic review and meta-analysis of observational and clinical investigations. J Diabetes Metab Disord. 2020, 19:575-584. 10.1007/s40200-019-00475-2

18. Biolato M, Manca F, Marrone G, et al.: Intestinal permeability after Mediterranean diet and low-fat diet in non-alcoholic fatty liver disease. World J Gastroenterol. 2019, 25:509-520. 10.3748/wjg.v25.i4.509

19. Gorzynik-Debicka M, Przychodzen P, Cappello F, et al.: Potential health benefits of olive oil and plant polyphenols. Int J Mol Sci. 2018, 19:686. 10.3390/ijms19030686

20. Abenavoli L, Boccuto L, Federico A, Dallio M, Loguercio C, Di Renzo L, De Lorenzo A: Diet and nonalcoholic fatty liver disease: the Mediterranean way. Int J Environ Res Public Health. 2019, 16:3011. 10.3390/ijerph16173011

21. Yamada N, Matsushima-Nishiwaki R, Masue A, Taguchi K, Kozawa O: Olive oil polyphenols suppress the TGF- $\alpha$-induced migration of hepatocellular carcinoma cells. Biomed Rep. 2019, 1:1-5.

22. Margini C, Dufour JF: The story of HCC in NAFLD: from epidemiology, across pathogenesis, to prevention and treatment. Liver Int. 2016, 36:317-324. 10.1111/liv.13031

23. Nediani C, Ruzzolini J, Romani A, Calorini L: Oleuropein, a bioactive compound from Olea europaea L., as a potential preventive and therapeutic agent in non-communicable diseases. Antioxidants (Basel). 2019, 8:578. 10.3390/antiox8120578

24. Choi Y, Lee JE, Chang Y, Kim MK, Sung E, Shin H, Ryu S: Dietary sodium and potassium intake in relation to non-alcoholic fatty liver disease. Br J Nutr. 2016, 116:1447-1456. 10.1017/S0007114516003391

25. Suckling RJ, Swift PA: The health impacts of dietary sodium and a low-salt diet . Clin Med (Lond). 2015, 15:585-588. 10.7861/clinmedicine.15-6-585 